\title{
Relationship of Climatic Factors with Incidence of Major Insect Pest of Teak Seedlings at Coastal Odisha, India
}

\author{
M.K. Tripathy", M. Rout and H.K. Das \\ College of Forestry, O.U.A.T., Bhubaneswar, India \\ *Corresponding author
}

\section{A B S T R A C T}

\section{Keywords \\ Teak defoliator, Teak \\ skeletoniser, Co- \\ relation, Seasonal coincidence}

Article Info

Accepted:

26 January 2018

Available Online:

10 February 2018
Teak Tectona grandis (Linnaeus), is the most preferred afforested species of Odisha as well as India. Among the total 42 species of insect pests recorded in seedlings of this sps., Hyblaea puera, Eutectona machaeralis, Lachnosterna serrata, five species of grass hoppers, Jassids, spittle bugs, Myllocerus beetle, Mealy bugs, aphid and, Tussock moth larvae were prominent in causing damage. The study further revealed that population of defoliator larvae was highest during $1^{\text {st }}$ fortnight of June and continued upto $1^{\text {st }}$ fortnight of December with peak appearance during $2^{\text {nd }}$ fortnight of July, 2016 (11.23 larvae/seedling). Grass hopper population ranged from 2.34 to 3.27 per seedling and that of skeletoniser was ranged from 0.45 to 3.07 with peak availability during September $2^{\text {nd }}$ fort night, 2016 (5.25 larvae/seedling). Aphid and other sucking pests made their appearance for a short period only but percentage plant mortality recorded due to white grub attack was ranged from minimum of $1.32 \%$ during September $1^{\text {st }}$ fortnight to $30.85 \%$ during July $1^{\text {st }}$ fortnight and the pest was appeared from June $1^{\text {st }}$ fort night to September $1^{\text {st }}$ fortnight only.

\section{Introduction}

Teak, Tectona grandis (Linnaeus), is one of the most preferred timber species of Odisha as well as India. In India, it grows naturally in 9 million hectares of area and considered as one of the top five tropical plantation species of the world. About 187 insect species have been found feeding on living teak tree in India which includes 78 species from order Lepidoptera, 40 species from order Coleoptera and 18 species from order Orthoptera (Hutacharern and Tubtim, 1995). In fact, the total number of insect pests infesting this plant might be more because of report of additional species by several workers around the country during subsequent years after the base line report. Instead of substantial damage, no such established management practice is available in this country because of lack of systematic study in many aspects of the pests including biology, ecological studies like population density fluctuation, factors governing host preference of individual pests etc. Moreover, for economic, technical and social reasons chemical control strategies in spite of their availabilities are not preferred by the growers. In some advance states like Kerala some private growers are coming forward for taking control operations against the major pests. Hence in the present investigation an attempt is being made for bringing out a systematic 
documentation regarding the damage caused by different insect pests attacking teak seedlings in the state as well as to find out the relationship of different climatic factors with their incidence.

\section{Materials and Methods}

Abundance of defoliating pests of teak was recorded at five different locations viz., Silviculuture Research Station, Bhubaneswar, College of Forestry, OUAT campus, Poplar field of College of Forestry, Live stocks Farm of OUAT and Taratua Hi-tech Nursery located in Khurda. At each location, plants were divided into 3 categories such as (i) Seedlings0-1 year old (ii) Plants- 1-3 year old (iii) Plants- more than 3 years old. For seedlings, all the leaves are selected from 30 plants and sampled from which leaves were counted for number of healthy and damaged leaves, thus percent infestation due to defoliation was worked out by using standard formula,

Number of healthy leaves Number of infested leaves

$\%$ defoliation $=$---------------------- x 100

Number of uninfested leaves

The population of defoliators viz., skeletonizer, defoliator and tussock moths etc. were expressed as no. of larvae/plant, Population of sucking insects like Aphids, Mealybug sand Cercopids were expressed as no. per top $10 \mathrm{~cm}$ shoot. Leaf hopper density was expressed as no. per leaf.

Leaves were sampled from top, middle and bottom of the plants. Observations were recorded at fortnightly interval along with major weather parameter viz., temperature, relative humidity and rainfall at each location. Based on these data, linear correlation was worked out as per Gomez and Gomez (1984) and identification of insect pests was made as per procedure of Patil et al., (2016).

\section{Results and Discussion}

The data presented in Table 1 revealed that total defoliation percentage was varied from $33.64 \%$ as recorded from $1^{\text {st }}$ fortnight of June, 2016 to $78.74 \%$ recorded during $2^{\text {nd }}$ fortnight of July, 2017.

Month of July and August, 2016 and July, 2017 recorded highest defoliation percentage of leaves which gradually lowered down from winter season onwards. From January $1^{\text {st }}$ fortnight to May $2^{\text {nd }}$ fortnight the defoliation percentage was maintained at minimum level and was ranged from $5.27 \%$ to $3.18 \%$ in seedlings of the studied locations.

Larval population of Hyblaea puera was varied from 0.34 larvae/seedlings (June $1^{\text {st }}$ fortnight) to 0.35larvae/seedlings (December $1^{\text {st }}$ fortnight) with the peak availability during July $\quad 2^{\text {nd }} \quad$ fortnight, $2016 \quad$ (11.23 larvae/seedlings). Second year study also followed the same trend. However, population of Eutectona mechaeralis was available throughout the year except the peak of summer at $2^{\text {nd }}$ fortnight of May, 2016, when critically low population was observed.

The population ranged from 0.45 larvae/seedling (June, 2016) to 3.07 larvae/seedling (July, 2017). The peak being observed during $2^{\text {nd }}$ fortnight of September, 2016 (5.26 larvae/seedling).

Data on Table 2 presented the population densities of other pests in teak seedlings during the observation period. Grasshopper population has varied from $2.34 /$ seedlings $\left(1^{\text {st }}\right.$ fortnight of June, 2016) to $3.27 /$ seedlings $\left(2^{\text {nd }}\right.$ fortnight of July, 2017). Mealy bugs made their appearance during June, 2016 and disappeared from July onwards again reappeared from $2^{\text {nd }}$ fortnight of December (6.35 numbers/top $10 \mathrm{~cm}$ shoot) which was continued till the end of June, 2017. Aphids 
population in seedlings were recorded from $2^{\text {nd }}$ fortnight of September, 2016 (20.35/ top $10 \mathrm{~cm}$ of shoot) and continued up to $2^{\text {nd }}$ fortnight of November, 2016 (15.35/top $10 \mathrm{~cm}$ shoot) and during 2017 aphids were observed during the month of July. Likewise tussock moth caterpillars which is another important polyphagous defoliator has made its appearance from $1^{\text {st }}$ fortnight of July, 2016 (1.85 no's/seedlings) and continued up to $2^{\text {nd }}$ fortnight of December, 2016. Spittle bug and jassid population was confined to kharif season only. The pentatomids appeared during the late Kharif season ( $2^{\text {nd }}$ fortnight of September, 2016) and continued till the end of the observation period except April and July, 2017. Myllocerus beetles, spittle bug and white grubs were recorded their presence during the initial period of rainy season. Highest percentage of plant mortality due to white grubs attack was recorded during $2^{\text {nd }}$ fortnight of July, 2016 (32.34\%) and minimum was recorded at $1^{\text {st }}$ fortnight of September, 2016 (1.32\%).

As observed, percentage peak defoliation was recorded during the month of July and August 2016 and second but small peak was recorded during October month of 2016. Hyblaea purea larval population density was observed from 1st fortnight of June, 2016 and June, 2017 which was very low and percentage defoliation was attributed by other species like Chrysomelids and Myllocerus beetle etc. However, in seedlings, population of other pests are mentioned in Table 1. Defoliator population showed a second but small peak during October 2nd fortnight (1.81/seedling) and continued to be available up to 1 st fortnight of December with low larval population. However, Skeletonizer larvae make its appearance throughout the year and from November to May, the population critically low. Peak larval population was noticed during 2nd fortnight of September, 2016 (5.26/seedling). Different earlier workers reported differently regarding the appearance of peak incidence from different places. Baksha and Crawley (1998) from Chittagong reported the peaks to be varied from one to three there during their experimental period from 1989-90 to 199293, i.e. during 3rd week of May (1989), 1st to 2nd week of June (1992).

Kabade et al., (2015) from Gujarat stated that appearance of teak skeletonizer and defoliator in their situation started appearing from April and June respectively and reached peak (>40\%) during July-August, declined during rest of the months.

Among other pests, Grasshopper population was available throughout the year expect January, February and March. Mealy bugs, aphids, jassids, spittle bugs, myllocerus beetles and pentatomids etc. were appeared for a single season only and their population density was not too high except for myllocerus beetles and their highest population (10.82/seedling) was recorded during 1st fortnight of July followed by 2nd fortnight of the same month. Workers like Pandey et al., (2010) from Uttar Pradesh also reported 12 numbers of Arthropods including green leaf hopper, cow bug, grasshopper, hadda beetle, red cotton bug infesting teak from their survey.

The value of correlation coefficient in between mean percentage defoliation due to all pests was significant and positive for percentage relative humidity recorded at 14 hour and rain fall whereas a non-significant but negative correlation was found between mean maximum temperature with mean percentage defoliation. Likely larval population densities of both the major defoliator species was positively correlated with percentage relative humidity recorded at 14 hour as well as rain fall which were statistically significant both at 5\% and 1\% level (Table 3 and Fig. 1 to 5). 
Table.1 Defoliation dynamics and larval population densities of major foliage feeding pests in teak seedling during the experimental period (June, 2016-July, 17) at Bhubaneswar

\begin{tabular}{|c|c|c|c|}
\hline \multirow{2}{*}{$\begin{array}{l}\text { Observation week } \\
\text { no. }\end{array}$} & \multirow{2}{*}{$\begin{array}{l}\text { Mean Percentage } \\
\text { defoliation }(\%)\end{array}$} & \multicolumn{2}{|c|}{ Mean Larval population number per seedling } \\
\hline & & H. puera & E. machaeralis \\
\hline June I & $\begin{array}{l}33.64 \pm 2.04 \\
(20.54-42.07)\end{array}$ & $0.34 \pm 0.60(0.00-2.00)$ & $0.45 \pm 0.47(0.00-1.00)$ \\
\hline June II & $\begin{array}{c}44.28 \pm 1.61 \\
(31.75-52.75)\end{array}$ & $1.36 \pm 1.08(0.00-3.00)$ & $0.63 \pm 0.35(0.00-2.00)$ \\
\hline July I & $\begin{array}{c}71.19 \pm 1.42 \\
(59.96-84.33)\end{array}$ & $7.87 \pm 1.09(2.00-15.00)$ & $0.23 \pm 0.16(0.00-1.00)$ \\
\hline July II & $\begin{array}{c}82.74 \pm 1.37 \\
(70.23-92.06)\end{array}$ & $11.23 \pm 2.03(4.00-18.00)$ & $0.36 \pm 0.24(0.00-1.00)$ \\
\hline Aug I & $\begin{array}{c}72.23 \pm 2.20 \\
(61.89-81.53)\end{array}$ & $6.28 \pm 2.86(2.00-12.00)$ & $1.74 \pm 1.08(0.00-3.00)$ \\
\hline Aug II & $\begin{array}{c}73.69 \pm 2.17 \\
(51.04-74.24)\end{array}$ & $5.87 \pm 2.57(1.00-8.00)$ & $3.67 \pm 1.05(1.00-6.00)$ \\
\hline Sept I & $\begin{array}{c}35.23 \pm 1.72 \\
(25.06-46.75)\end{array}$ & $1.50 \pm 1.16(0.00-4.00)$ & $4.76 \pm 2.05(2.00-8.00)$ \\
\hline Sept II & $\begin{array}{c}32.49 \pm 4.01 \\
(21.35-47.75)\end{array}$ & $1.27 \pm 0.85(0.00-3.00)$ & $5.26 \pm 2.46(1.00-6.00)$ \\
\hline Oct I & $\begin{array}{c}54.68 \pm 4.58 \\
(48.90-63.56)\end{array}$ & $1.58 \pm 1.04(0.00-3.00)$ & $3.64 \pm 1.37(0.00-4.00)$ \\
\hline Oct II & $\begin{array}{c}67.34 \pm 6.35 \\
(60.54-73.71)\end{array}$ & $1.81 \pm 3.24(0.00-3.00)$ & $0.44 \pm 0.32(0.00-2.00)$ \\
\hline Nov I & $\begin{array}{c}16.32 \pm 6.15 \\
(12.34-20.21)\end{array}$ & $0.42 \pm 0.43(0.00-2.00)$ & $0.21 \pm 0.20(0.00-1.00)$ \\
\hline Nov II & $\begin{array}{c}16.75 \pm 6.43 \\
(13.58-19.87)\end{array}$ & $0.32 \pm 0.34(0.00-1.00)$ & $0.12 \pm 0.15(0.00-1.00)$ \\
\hline Dec I & $\begin{array}{c}15.34 \pm 5.51 \\
(12.32-19.67)\end{array}$ & $0.35 \pm 0.37(0.00-1.00)$ & $0.42 \pm 0.41(0.00-1.00)$ \\
\hline Dec II & $\begin{array}{l}8.78 \pm 3.31 \\
(6.47-9.57)\end{array}$ & 0.00 & $0.54 \pm 0.52(0.00-2.00)$ \\
\hline $\operatorname{Jan} I$ & $\begin{array}{l}5.27 \pm 7.15 \\
(2.81-8.40)\end{array}$ & 0.00 & $0.46 \pm 0.38(0.00-2.00)$ \\
\hline Jan II & $\begin{array}{c}5.84 \pm 2.8 \\
(3.78-9.83)\end{array}$ & 0.00 & $0.38 \pm 0.41(0.00-2.00)$ \\
\hline Feb I & $\begin{array}{l}4.21 \pm 2.49 \\
(2.07-8.73)\end{array}$ & 0.00 & $0.27 \pm 0.23(0.00-1.00)$ \\
\hline Feb II & $\begin{array}{l}3.57 \pm 3.24 \\
(0.00-8.29)\end{array}$ & 0.00 & $0.15 \pm 0.17(0.00-1.00)$ \\
\hline Mar I & $\begin{array}{l}2.51 \pm 2.34 \\
(0.00-8.43)\end{array}$ & 0.00 & $0.34 \pm 0.25(0.00-1.00)$ \\
\hline Mar II & $\begin{array}{l}3.54 \pm 4.32 \\
(0.00-7.35)\end{array}$ & 0.00 & $0.52 \pm 0.47(0.00-2.00)$ \\
\hline Apr I & $\begin{array}{l}4.25 \pm 3.87 \\
(0.00-8.21)\end{array}$ & 0.00 & $0.69 \pm 0.41(0.00-2.00)$ \\
\hline Apr II & $\begin{array}{l}2.14 \pm 2.11 \\
(0.00-4.65)\end{array}$ & 0.00 & $0.81 \pm 0.64(0.00-3.00)$ \\
\hline May I & $\begin{array}{c}2.36 \pm 1.64 \\
(0.00-5.32)\end{array}$ & 0.00 & $0.87 \pm 0.67(0.00-3.00)$ \\
\hline May II & $\begin{array}{l}3.18 \pm 1.86 \\
(0.00-5.12)\end{array}$ & 0.00 & $0.79 \pm 0.78(0.00-3.00)$ \\
\hline June I & $\begin{array}{c}20.64 \pm 2.04 \\
(15.54-36.27)\end{array}$ & $0.28 \pm 0.32(0.00-2.00)$ & $0.36 \pm 0.27(0.00-1.00)$ \\
\hline June II & $\begin{array}{c}39.78 \pm 1.76 \\
(21.45-64.75)\end{array}$ & $1.06 \pm 1.08(0.00-3.00)$ & $0.75 \pm 0.35(0.00-2.00)$ \\
\hline July I & $\begin{array}{c}82.19 \pm 1.56 \\
(59.96-84.33)\end{array}$ & $8.36 \pm 2.09(2.00-15.00)$ & $2.14 \pm 1.08(0.00-3.00)$ \\
\hline July II & $\begin{array}{c}78.74 \pm 2.37 \\
(45.86-90.66)\end{array}$ & $12.03 \pm 2.03(3.00-18.00)$ & $3.07 \pm 1.05(1.00-6.00)$ \\
\hline
\end{tabular}

*Each figure denotes Mean $\pm S . D$, figures in parenthesis are range values. 
Table.2 Infestation of other insect pests in teak seedlings at Bhubaneswar during the observation period (2016-17) at Bhubaneswar

\begin{tabular}{|c|c|c|c|c|c|c|c|c|c|}
\hline $\begin{array}{l}\text { Observati } \\
\text { on weeks }\end{array}$ & $\begin{array}{l}\text { No. of grasshopper } \\
\text { (nymphs \& adults) } \\
\text { per plant }\end{array}$ & $\begin{array}{l}\text { No. of mealy } \\
\text { bugs per top } 10 \\
\text { cm shoot }\end{array}$ & $\begin{array}{l}\text { No. of aphids } \\
\text { per top } 10 \mathrm{~cm} \\
\text { shoot }\end{array}$ & $\begin{array}{l}\text { No. of } \\
\text { lymantrids per } \\
\text { plant }\end{array}$ & $\begin{array}{l}\text { No. of jassids per } \\
3 \text { leaves }\end{array}$ & $\begin{array}{l}\text { No. of spittle } \\
\text { bug \& nymphs } \\
\text { per plant }\end{array}$ & $\begin{array}{l}\text { No. of pentatomid (bugs } \\
\& \text { nymphs) per plant }\end{array}$ & $\begin{array}{l}\text { No. of myllocerus } \\
\text { bettle (adults) per } \\
\text { plant }\end{array}$ & $\begin{array}{l}\text { Percentage plant } \\
\text { mortality due to } \\
\text { white grubs }\end{array}$ \\
\hline June I & $2.34(1.00-3.00)$ & $\begin{array}{l}15.35(0.00- \\
25.00)\end{array}$ & 0.00 & 0.00 & $2.35(0.00-6.00)$ & 0.00 & 0.00 & $7.67(1.00-8.00)$ & $24.47(2.56-23.56)$ \\
\hline June II & $2.78(1.00-3.00)$ & $\begin{array}{l}16.55(0.00- \\
28.00)\end{array}$ & 0.00 & 0.00 & $1.85(0.00-6.00)$ & $\begin{array}{l}3.11 \\
(0.00-12.00)\end{array}$ & 0.00 & $8.32(1.00-10.00)$ & $25.08(3.56-26.56)$ \\
\hline July I & $3.65(1.00-5.00)$ & 0.00 & 0.00 & $1.85(0.00-3.00)$ & $3.45(0.00-7.00)$ & $4.37(0.00-14.00)$ & 0.00 & $10.82(2.00-12.00)$ & $30.85(4.26-32.64)$ \\
\hline July II & $3.43(1.00-5.00)$ & 0.00 & 0.00 & $2.65(0.00-4.00)$ & $3.85(0.00-8.00)$ & $4.5(0.00-12.00)$ & 0.00 & $9.53(1.00-10.00)$ & $32.34(3.26-35.64)$ \\
\hline Aug I & $3.12(0.00-5.00)$ & 0.00 & 0.00 & $1.25(0.00-3.00)$ & $2.15(0.00-4.00)$ & $3.57(0.00-6.00)$ & 0.00 & $6.71(0.00-8.00)$ & $4.32(0.00-7.87)$ \\
\hline Aug II & $2.73(0.00-4.00)$ & 0.00 & 0.00 & $0.85(0.00-3.00)$ & $1.65(0.00-3.00)$ & $2.17(0.00-6.00)$ & 0.00 & $3.49(0.00-6.00)$ & $2.12(0.00-8.64)$ \\
\hline Sept I & $2.86(0.00-5.00)$ & 0.00 & 0.00 & $1.05(0.00-3.00)$ & 0.00 & $1.45(0.00-6.00)$ & 0.00 & 0.00 & $1.32(0.00-5.64)$ \\
\hline Sept II & $1.68(0.00-3.00)$ & 0.00 & $\begin{array}{l}20.35(0.00- \\
42.00)\end{array}$ & $0.65(0.00-3.00)$ & 0.00 & $1.03(0.00-3.00)$ & $1.37(0.00-3.00)$ & 0.00 & 0.00 \\
\hline Oct I & $1.47(0.00-3.00)$ & 0.00 & $\begin{array}{l}45.25(0.00- \\
64.00)\end{array}$ & $0.55(0.00-2.00)$ & 0.00 & 0.00 & $2.76(0.00-5.00)$ & 0.00 & 0.00 \\
\hline Oet II & $1.06(0.00-3.00)$ & 0.00 & $\begin{array}{l}52.15(0.00- \\
76.00)\end{array}$ & $0.45(0.00-2.00)$ & 0.00 & 0.00 & $2.59(0.00-6.00)$ & 0.00 & 0.00 \\
\hline Nov I & $1.28(0.00-3.00)$ & 0.00 & $\begin{array}{l}25.45(0.00- \\
36.00)\end{array}$ & $0.85(0.00-3.00)$ & 0.00 & 0.00 & $5.43(0.00-8.00)$ & 0.00 & 0.00 \\
\hline Nov II & $0.76(0.00-2.00)$ & 0.00 & $\begin{array}{l}15.25(0.00- \\
27.00)\end{array}$ & $0.73(0.00-2.00)$ & 0.00 & 0.00 & $7.80(0.00-12.00)$ & 0.00 & 0.00 \\
\hline$\overline{\text { Dec I }}$ & $0.48(0.00-2.00)$ & 0.00 & 0.00 & $0.52(0.00-4.00)$ & 0.00 & 0.00 & $8.00(0.00-12.00)$ & 0.00 & 0.00 \\
\hline Dec II & $0.23(0.00-1.00)$ & 0.00 & 0.00 & $0.70(0.00-3.00)$ & 0.00 & 0.00 & $6.43(0.00-9.00)$ & 0.00 & 0.00 \\
\hline Jan I & 0.00 & $7.35(0.00-14.00)$ & 0.00 & 0.00 & $0.35(0.00-2.00)$ & 0.00 & $3.25(0.00-6.00)$ & 0.00 & 0.00 \\
\hline Jan II & 0.00 & $6.75(0.00-13.00)$ & 0.00 & 0.00 & $0.45(0.00-2.00)$ & 0.00 & $2.54(0.00-6.00)$ & 0.00 & 0.00 \\
\hline Feb I & 0.00 & $5.55(0.00-11.00)$ & 0.00 & 0.00 & 0.00 & 0.00 & $2.65(0.00-5.00)$ & 0.00 & 0.00 \\
\hline Feb II & 0.00 & $6.35(0.00-12.00)$ & 0.00 & 0.00 & 0.00 & 0.00 & $3.45(0.00-7.00)$ & 0.00 & 0.00 \\
\hline Mar I & 0.00 & $7.45(0.00-15.00)$ & 0.00 & 0.00 & 0.00 & 0.00 & $0.45(0.00-2.00)$ & $2.47(0.00-5.00)$ & 0.00 \\
\hline Mar II & 0.00 & $6.25(0.00-15.00)$ & 0.00 & 0.00 & 0.00 & 0.00 & $0.49(0.00-2.00)$ & $2.13(0.00-5.00)$ & 0.00 \\
\hline Apr I & $4.23(1.00-5.00)$ & $\begin{array}{l}18.55(0.00- \\
28.00)\end{array}$ & 0.00 & 0.00 & 0.00 & 0.00 & 0.00 & $3.28(0.00-6.00)$ & 0.00 \\
\hline Apr II & $3.17(0.00-5.00)$ & $\begin{array}{l}20.35(0.00- \\
28.00)\end{array}$ & 0.00 & 0.00 & 0.00 & 0.00 & 0.00 & $4.63(0.00-6.00)$ & 0.00 \\
\hline May I & $2.64(0.00-4.00)$ & $\begin{array}{l}20.65(0.00- \\
25.00)\end{array}$ & 0.00 & 0.00 & 0.00 & 0.00 & $3.49(0.00-6.00)$ & $3.64(0.00-6.00)$ & 0.00 \\
\hline May II & $2.14(0.00-4.00)$ & $\begin{array}{l}16.75(0.00- \\
26.00)\end{array}$ & 0.00 & 0.00 & 0.00 & 0.00 & $2.76(0.00-5.00)$ & $4.73(0.00-6.00)$ & 0.00 \\
\hline Jun I & $2.86(0.00-4.00)$ & $\begin{array}{l}12.25(0.00- \\
22.00)\end{array}$ & 0.00 & 0.00 & 0.00 & 0.00 & $0.55(0.00-2.00)$ & $5.31(0.00-6.00)$ & 0.00 \\
\hline Jun II & $2.54(0.00-5.00)$ & $\begin{array}{l}10.15(0.00- \\
18.00)\end{array}$ & 0.00 & 0.00 & 0.00 & 0.00 & $0.25(0.00-1.00)$ & $5.67(0.00-7.00)$ & 0.00 \\
\hline Jul I & $2.75(1.00-5.00)$ & 0.00 & 0.00 & $1.55(0.00-3.00)$ & $2.75(0.00-7.00)$ & $2.72(0.00-14.00)$ & 0.00 & $8.22(0.00-12.00)$ & $25.85(3.66-36.54)$ \\
\hline Jul II & $3.27(1.00-5.00)$ & 0.00 & 0.00 & $2.35(0.00-4.00)$ & $\begin{array}{l}3.45 \\
(0.00-8.00)\end{array}$ & $2.54(0.00-12.00)$ & 0.00 & $9.46(1.00-10.00)$ & $30.36(4.36-38.35)$ \\
\hline
\end{tabular}

Figures in parenthesis are range values 
Table.3 Correlation coefficient of major defoliating larval population and percentage defoliation in teak with weather parameters at Bhubaneswar (2016-2017)

\begin{tabular}{|c|c|c|c|c|}
\hline \multirow{2}{*}{\multicolumn{2}{|c|}{ Weather parameters }} & \multirow{2}{*}{$\begin{array}{l}\text { Mean \% } \\
\text { defoliation }\end{array}$} & \multicolumn{2}{|c|}{ Larval population } \\
\hline & & & Hyblaea purea & Eutectona machaeralis \\
\hline \multirow{2}{*}{$\begin{array}{l}\% \text { relative } \\
\text { humidity }\end{array}$} & $7 \mathrm{hr}$ & $0.368 \mathrm{NS}$ & $0.361 \mathrm{NS}$ & $0.371 \mathrm{NS}$ \\
\hline & 14hr & $0.860 * *$ & $0.659 * *$ & $0.623 * *$ \\
\hline \multicolumn{2}{|c|}{ Rain fall } & $0.889 * *$ & $0.758 * *$ & $0.541 * *$ \\
\hline \multicolumn{2}{|c|}{$\begin{array}{l}\text { Mean maximum } \\
\text { temperature }\end{array}$} & $-0.296 \mathrm{NS}$ & $-0.257 \mathrm{NS}$ & $-0.211 \mathrm{NS}$ \\
\hline \multicolumn{2}{|c|}{$\begin{array}{l}\text { Mean minimum } \\
\text { temperature }\end{array}$} & $0.455 * *$ & $0.359 \mathrm{NS}$ & $0.355 \mathrm{NS}$ \\
\hline
\end{tabular}

*-significant at $5 \%$ level, **-Significant at $1 \%$ level
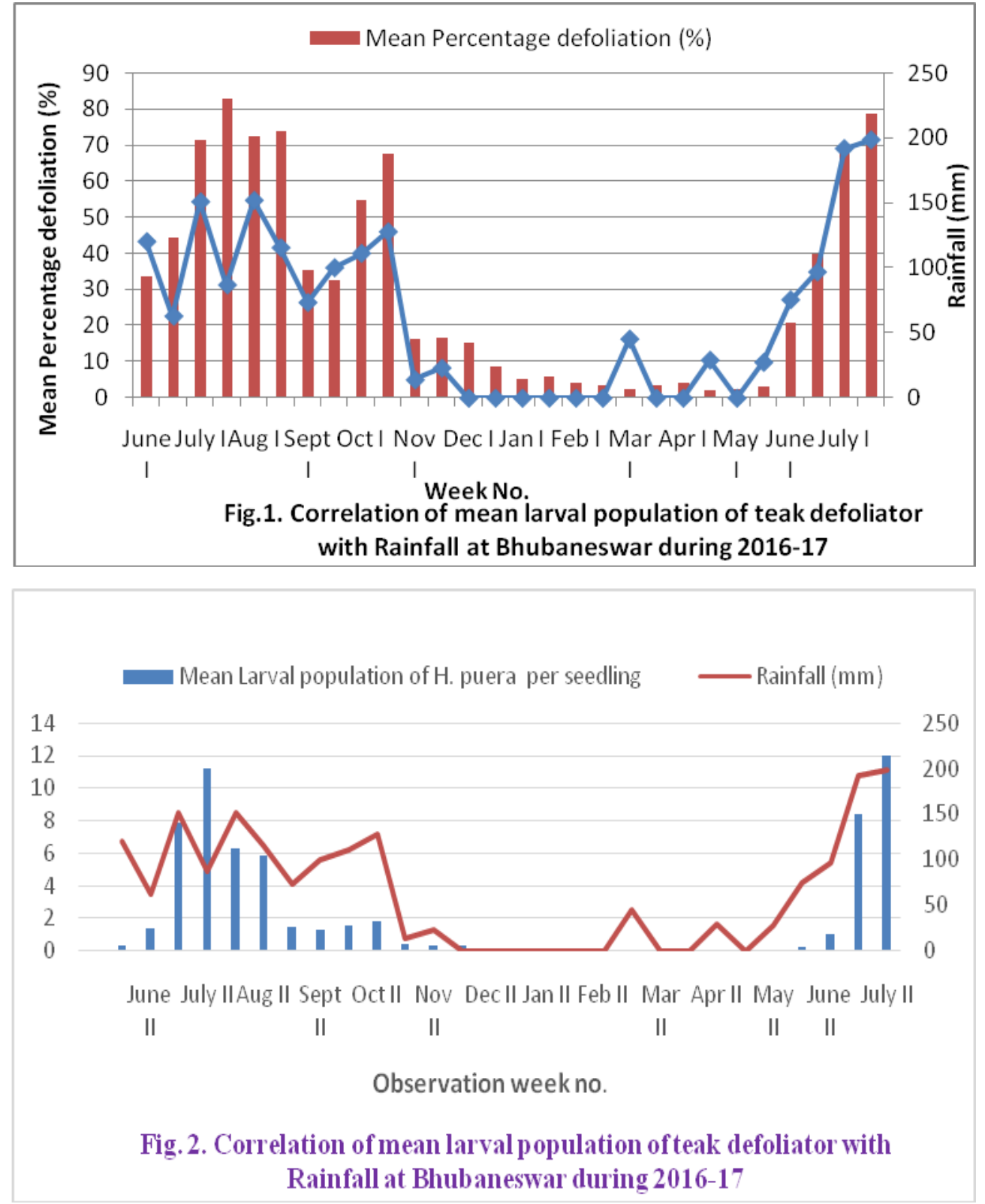


\section{Mean Larval population of E. machaeralis per seedling}

- Rainfall (mm)

6

5

4

3

2
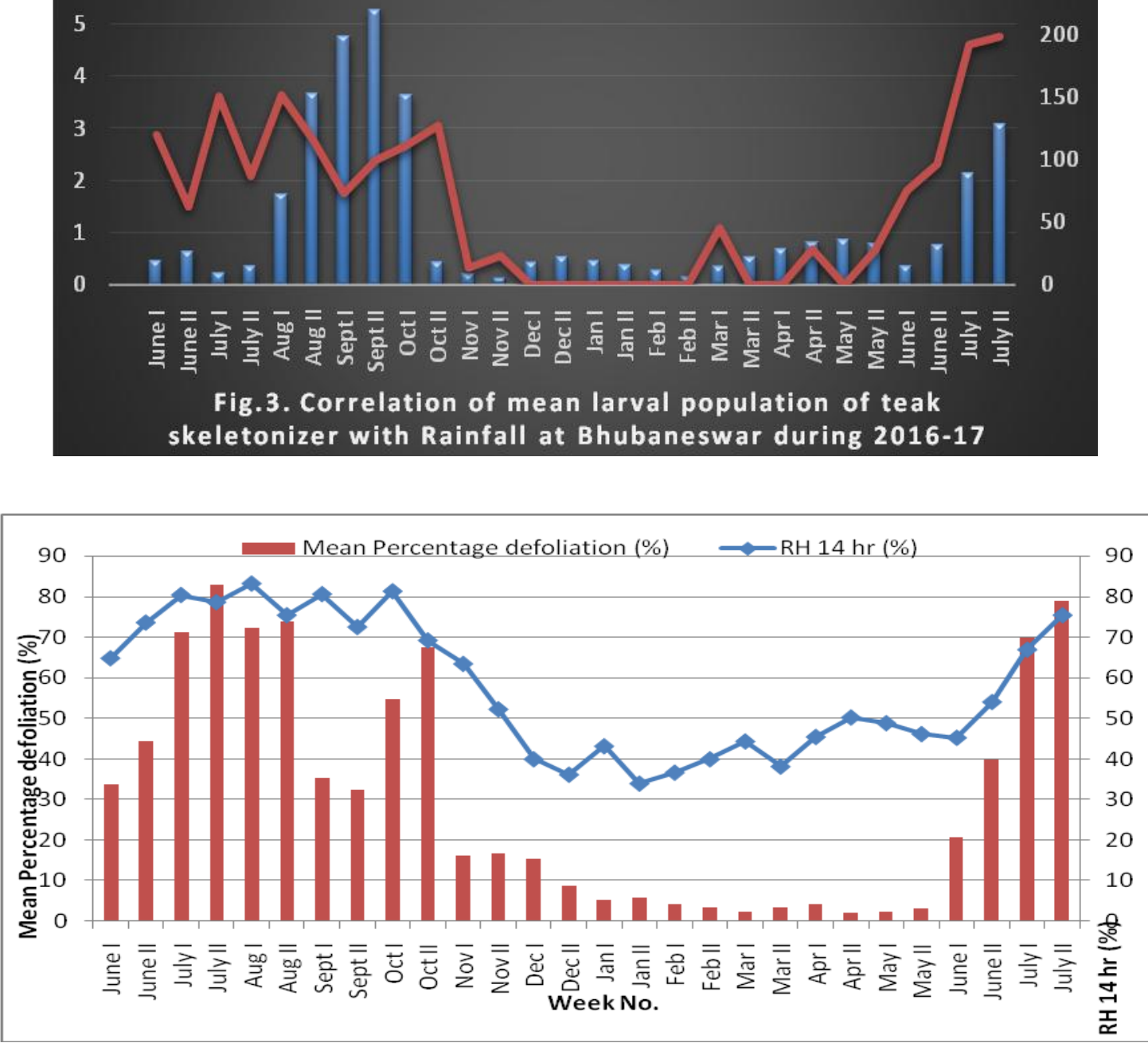

Fig.4. Correlation of mean larval population of teak defoliator with RH 14 hrs. (\%) at Bhubaneswar

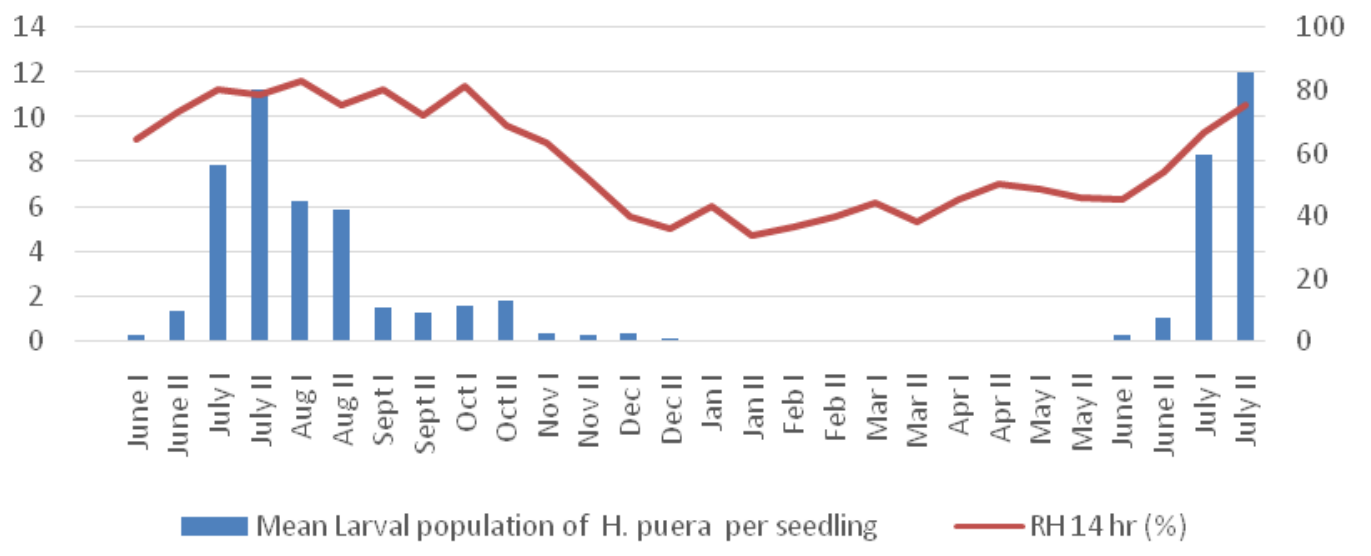




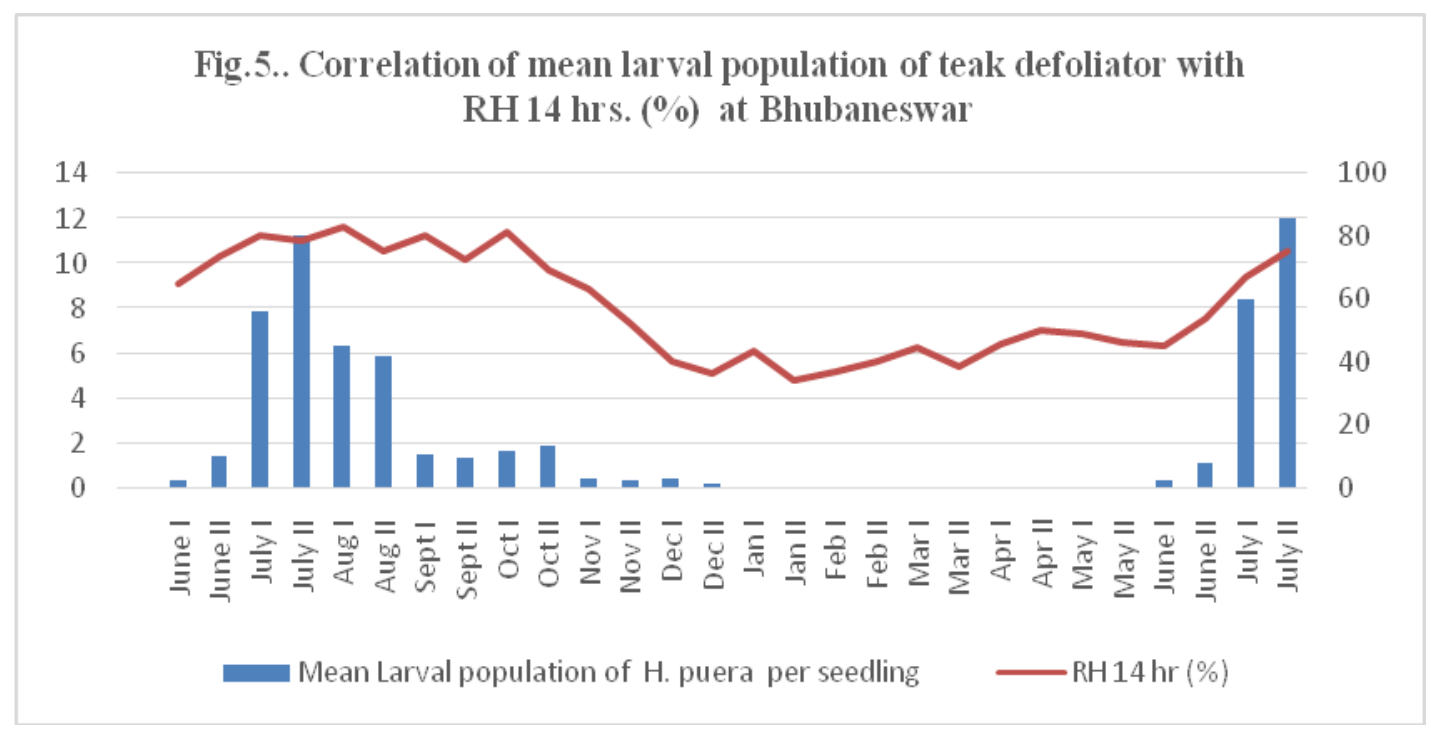

Hence, it can be concluded from the present experiment that $H$. purea, E. mecharalis, white grubs and Myllocerus beetles are the main insect pest of teak seedlings at coastal region of Odisha and it is required to take appropriate management strategies at proper time for these pests

\section{References}

Baksha, MW and Crawley MJ. 1998. Population dynamics of teak defoliator, Hyblaea puera Cram. (Lep., Hyblaeidae) in teak plantations of Bangladesh, Journal of Applied Entomology, 122(1- 5) :79-83

Gomez, KA and Gomez, AA. 1984. Statistical procedure for agricultural research, John Wiley and Sons, 1-645.

Hutacharern, C and Tubtim, N. 1995. Checklist of forest insects in Thailand,
Bangkok: Office of Environmental Policy and Planning, Ministry of Science, Technology and Environment. Kabade, KH, Patel ZP and Gawali ST. 2015. Crop-pest map in teak against teak skeletonizer and defoliator under heavy rainfall zone of south Gujarat condition, Plant Archives, 15(1): 101-103.

Pandey, V. Singh, A.K. and Sharma R.P. 2010. Biodiversity of insect pests associated with teak (Tectona grandis Lf) in Eastern Uttar Pradesh of India, Research Journal of Forestry, 4(3): 136-144.

Patil, S.S, Sutar., MV and Sathe TV. 2016. Diversity, Biology and Control of Insect pests of teak Tectona grandis (Linnaeus) from western Maharashtra, Bio Life, 4(1): 141-146.

\section{How to cite this article:}

Tripathy, M.K., M. Rout and Das, H.K. 2018. Relationship of Climatic Factors with Incidence of Major Insect Pest of Teak Seedlings at Coastal Odisha. Int.J.Curr.Microbiol.App.Sci. 7(02): 3144-3151. doi: https://doi.org/10.20546/ijcmas.2018.702.378 